\title{
A Single Column Gas Chromatographic Method for the Direct Trace Analysis of High Boiling Components in Rums ${ }^{1}$
}

\author{
Heriberto Batiz and Eduardo Rosado ${ }^{2}$
}

\begin{abstract}
A simple single-pass gas chromatographic method is described for identifying and determining the concentration of congeners not previously reported in commercial Puerto Rican rums. It makes use of well-stabilized packed highefficiency analytical columns to afford rapid separation of about 23 new congeners, of which eight were positively identified. More than 43 congeners were separated with the same column operated at two different carrier flows, when used to analyze samples of 1976 Puerto Rican commercial rums.
\end{abstract}

\section{INTRODUCTION}

Direct analytical packed column gas chromatographic analyses of components present in distilled alcoholic beverages, such as whisky, brandy, and rum, have been done almost since the advent of this unique technique $(2,3)$. Although many high-efficiency packed columns are available for this purpose, when direct analysis of distilled alcoholic beverages is required, only a limited number of components may be determined in a single separation $(1,4)$. This is because not more than 10 components are at sufficiently high concentration for adequate separation and quantitative measurement. In order to carry out a comprehensive analysis, concentration procedures must be employed followed by selective extraction of desirable components $(5,6)$ or by utilizing methods based on preparative capillary techniques (1). Direct single-pass trace analysis in the original sample without pretreatment is difficult. The situation here is not lack of detectors sensitive enough, but rather problems connected with high liquid phase bleeding of the columns at high temperatures and interference to a low level signal to noise ratio caused by carrier flow. The development of sufficiently stabilized columns may allow the use of a lower chromatographic attenuation level and detection of additional high boiling components.

The purpose of the present paper is to discuss the preparation and evaluation of suitable high-efficiency analytical packed columns, based on Carbowax $20 \mathrm{M}^{3}$ as liquid phase. These columns after stabilization, are

${ }^{1}$ Manuscript submitted to Editorial Board July 26, 1977.

${ }^{2}$ Chemist and Research Assistant, respectively, Rum Pilot Plant, Agricultural Experiment Station, College of Agricultural Sciences, Mayagüez Campus, University of Puerto Rico, Río Piedras, P.R.

${ }^{3}$ Trade names are used in this publication solely for the purpose of providing specific information. Mention of a trade name does not constitute a guarantee or warranty of equipment or materials by the Agricultural Experiment Station of the University of Puerto Rico or an endorsement over other equipment or materials not mentioned. 
exceptionally efficient for trace analysis of the high boiling components present in distilled alcoholic beverages. The method developed is intended for the scanning of the composition of the sample; for the estimation of the concentration of the minor high boiling components which separate after the isoamyl alcohol signal in Carbowax 20M; and for the identification of the origin of distilled alcoholic beverages. Results with selected alcoholic beverages are given along with identification of several new important congeners, and a comprehensive analysis of $1976 \mathrm{com}$ mercial Puerto Rican rums.

TABLE 1.-Retention times and composition of standard samples P-27 and P-31

\begin{tabular}{|c|c|c|c|c|}
\hline \multirow[t]{2}{*}{ Component } & \multicolumn{2}{|c|}{$\begin{array}{l}\text { Standard P-27 } \\
\text { (Carrier flow } \\
12 \mathrm{ml} / \mathrm{min} \text { ) }\end{array}$} & \multicolumn{2}{|c|}{$\begin{array}{l}\text { Standard P-31 } \\
\text { (Carrier flow } \\
25 \mathrm{ml} / \mathrm{min} \text { ) }\end{array}$} \\
\hline & Retention & Conc. & Retention & Conc. \\
\hline & Sec, & $\mathrm{Mg} / 100 \mathrm{ml}$ & Sec. & $\mathrm{Mg} / 100 \mathrm{ml}$ \\
\hline Acetaldehyde & 191 & 2.6 & - & - \\
\hline Methyl acetate & 260 & 1.3 & - & - \\
\hline Ethyl Acetate & 330 & 6.7 & - & - \\
\hline Acetal & 352 & 1.3 & - & - \\
\hline Propyl alcohol & 637 & 12.0 & - & - \\
\hline Isobutyl alcohol & 710 & 4.9 & - & - \\
\hline Isoamyl acetate & 750 & 2.7 & - & - \\
\hline Butyl alcohol & 780 & 14.2 & - & - \\
\hline Isoamyl alcohol & 840 & 18.6 & 551 & - \\
\hline Ethyl caproate & 879 & 4.9 & 576 & 0.38 \\
\hline Hexanol & - & - & 688 & .76 \\
\hline Ethyl caprylate & - & - & 841 & .38 \\
\hline Furfural & - & - & 884 & .76 \\
\hline Furfuryl acetate & - & - & 1,038 & .38 \\
\hline Octanol & - & - & 1,068 & .38 \\
\hline Ethyl caprate & - & - & 1,450 & .38 \\
\hline Ethyl laureate & - & - & 3,150 & .38 \\
\hline
\end{tabular}

\section{MATERIALS AND METHODS}

The work was accomplished with a Hewlet Packard 5754 B gas chromatograph equipped with a 3370 integrator. The required analytical columns were prepared at the Rum Pilot Plant. These colunns contained acid washed 60/80 mesh Chromosorb W coated with $5 \%$ Carbowax 20M, in several lengths of $3.2 \mathrm{~mm}$ o.d. stainless steel tubing. These tubings were cleaned in succession with chloroform, acetone, water, ammonia, water, nitric acid, water, ammonia, water, acetone, and methylene chloride, and then dried at $200^{\circ} \mathrm{C}$, by passing a slow flow of helium. To prepare the stationary phase, the volume of $30 \mathrm{~g}$ of solid support (sufficient to fill four of the tubings) was previously measured. The required amount of Carbowax 20M was weighed, dissolved in spectral grade methylene chloride, twice in volume of the solid support, and placed in a 
large stainless steel pot. The solid support was added immediately, a little at a time, with constant mixing to assure uniform coating of the solid phase. The mixing procedure was continued uninterruptedly, while direct heat was applied with an infrared lamp until the residual methylene chloride was evaporated. Finally, the material was introduced in the dried tubings with the help of an electric vibrator to get a uniform packing. All the packed columns were conditioned for $24 \mathrm{~h}$ at a carrier flow of 4

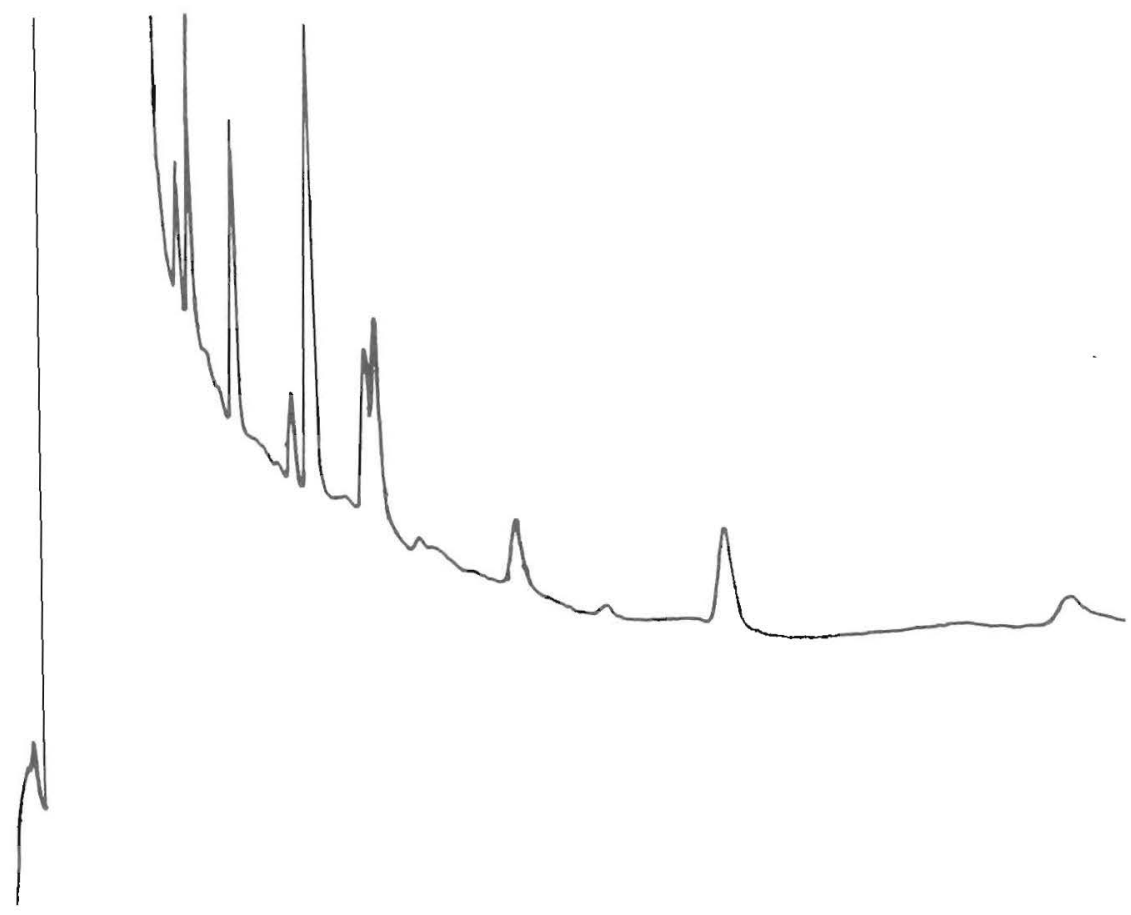

FrG. 1.-Chromatogram of standard sample P-31, $3 \mu \mathrm{l}$ in a high efficiency analytical packed column chromatogram; column PPR-132, $6.0 \mathrm{~m} \times 3.2 \mathrm{~mm}$ o.d. packed with acid washed, 60/80 mesh Chromosorb W coated with $5 \%$ Carbowax $20 \mathrm{M}$ carrier flow $25 \mathrm{ml} / \mathrm{min}$; initial temperature $58^{\circ} \mathrm{C}$ and final temperature $140^{\circ} \mathrm{C}$.

$\mathrm{ml} / \mathrm{min}$ and $140^{\circ} \mathrm{C}$, and for several days at a carrier flow of $15-20 \mathrm{ml} / \mathrm{min}$ and $150^{\circ} \mathrm{C}$. The columns were then kept at a carrier flow of $8 \mathrm{ml} / \mathrm{min}$ and $60^{\circ} \mathrm{C}$ until the signal base line of the recorder remained uniform when the chromatograph was programmed from 60 to $140^{\circ}$ C. The following seven columns were made and evaluated:

Analytical column Grams of support

PPR-126

PPR-127
Tubing length 


$\begin{array}{lll}\text { PPR-128 } & 6.9 & 5.5 \\ \text { PPR-129 } & 5.8 & 4.9 \\ \text { PPR-130 } & 7.2 & 5.5 \\ \text { PPR-131 } & 6.5 & 5.0 \\ \text { PPR-132 } & 8.2 & 6.0\end{array}$

For the final analyses columns PPR-130 and PPR-132 were selected. After appropriate working conditions for the columns were attained, the

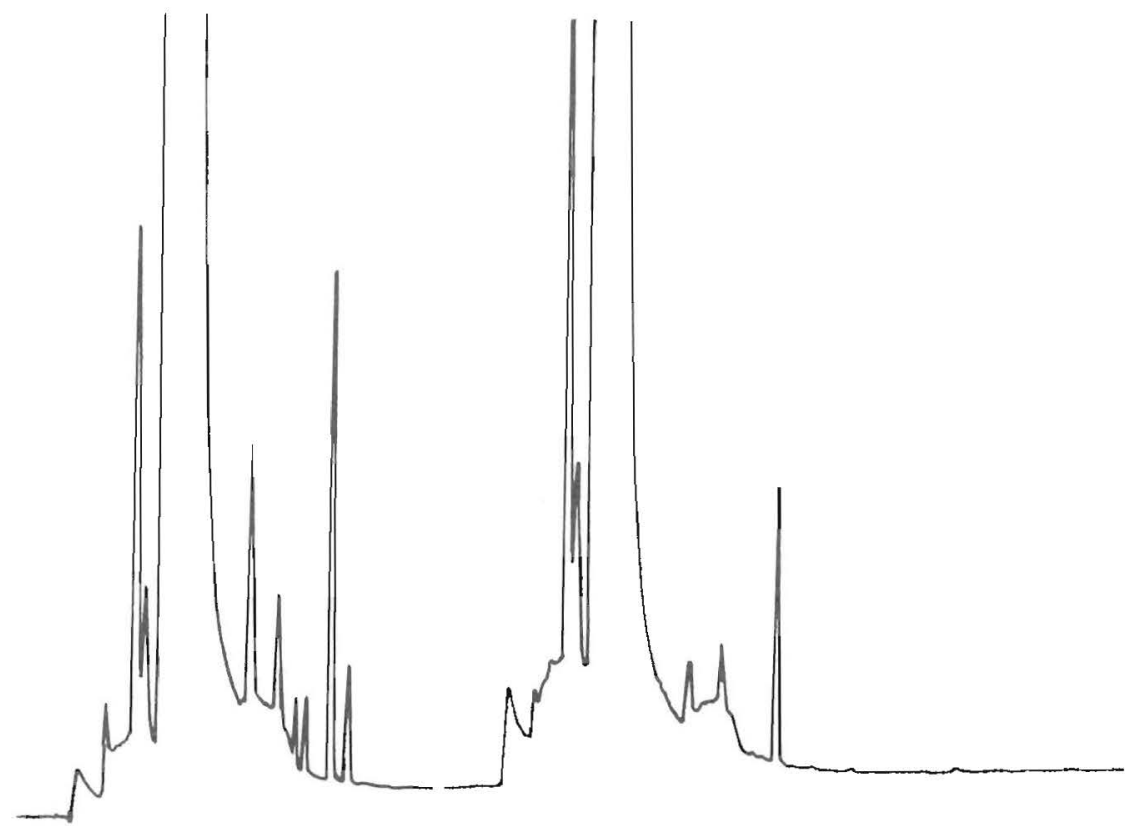

FIG. 2.-Chromatogram of standard sample P-27 and of R-703 $3 \mu l$ in a high efficiency analytical packed column chromatogram; column PPR-130, $5.5 \mathrm{~m} \times 3.2 \mathrm{~mm}$ o.d. packed with acid washed, $60 / 80$ mesh Chromosorb $\mathrm{W}$ coated with $5 \%$ Carbowax $20 \mathrm{M}$; carrier flow $12 \mathrm{ml} / \mathrm{min}$; initial temperature $50^{\circ} \mathrm{C}$ and final temperature $140^{\circ} \mathrm{C}$.

study was conducted by adjusting the different conditions of the chromatograph until the signal base line was completely stable at a sensitivity consistent both with the sample to be tested and with the bleeding rate of the column. The best chromatographic conditions obtained in this manner are given below:

\section{Optimum chromatographic conditions}

Chromatograph identification

Carrier flow $(\mathrm{He})$

Carrier pressure
HP-5754B

12 and $25 \mathrm{ml} / \mathrm{min}$

$7.0 \mathrm{~kg} / \mathrm{min}$ 
Oven temperature

FID temperature

TCD temperature

Injection temperature

Backflow valve temperature

Backflow valve flow (reversed)

Temperature program

Initial
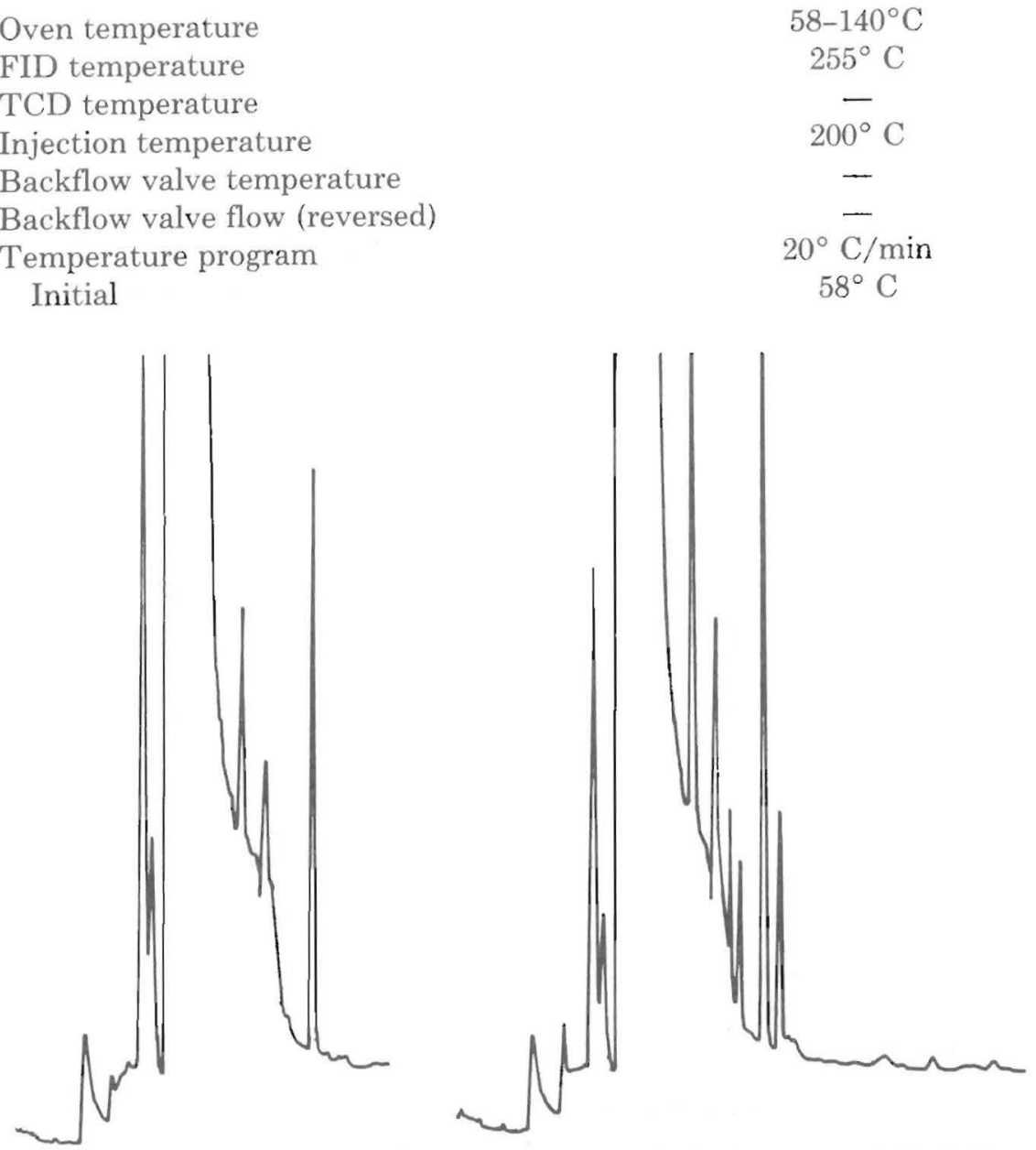

FIG. 3.-Chromatogram of R-704 and standard sample P-27, $3 \mu \mathrm{l}$ in a high efficiency analytical packed column chromatogram; column PPR-130, $5.5 \mathrm{~m} \times 3.2 \mathrm{~mm}$ o.d. packed with acid washed, 60/80 mesh Chromosorb W coated with $5 \%$ Carbowax $20 \mathrm{M}$; carrier flow $12 \mathrm{ml} / \mathrm{min}$; initial temperature $58^{\circ} \mathrm{C}$ and final temperature $140^{\circ} \mathrm{C}$.

\section{Final}

Post-injection interval

Recorder speed

Attentuation $140^{\circ} \mathrm{C}$

$3 \mathrm{~min}$.

$6.3 \mathrm{~mm} / 4 \mathrm{~min}$

$\times 40$

For this analysis, a three-microliter ( $\mu \mathrm{l})$ syringe injection of each sample was introduced in the column operated at the proper carrier flow. For high boiling congeners, the PPR-132 column was used, set at $25 \mathrm{ml} / \mathrm{min}$, 
and after the signal base line returned to base line, attenuated at 80 or 40 , the separation was allowed until all the components eluted. At least three successive separations of the same sample were obtained in this manner to get reproducible chromatograms. The column was then cleaned with spectral grade $80^{\circ} \mathrm{P}$ ethanol before proceeding with analysis of another sample. For the analysis of the more volatile congeners than isoamyl alcohol, a three $\mu$ l syringe injection of each sample was separated with the PPR-130 column, but operated at a carrier flow of $12 \mathrm{ml} / \mathrm{min}$.

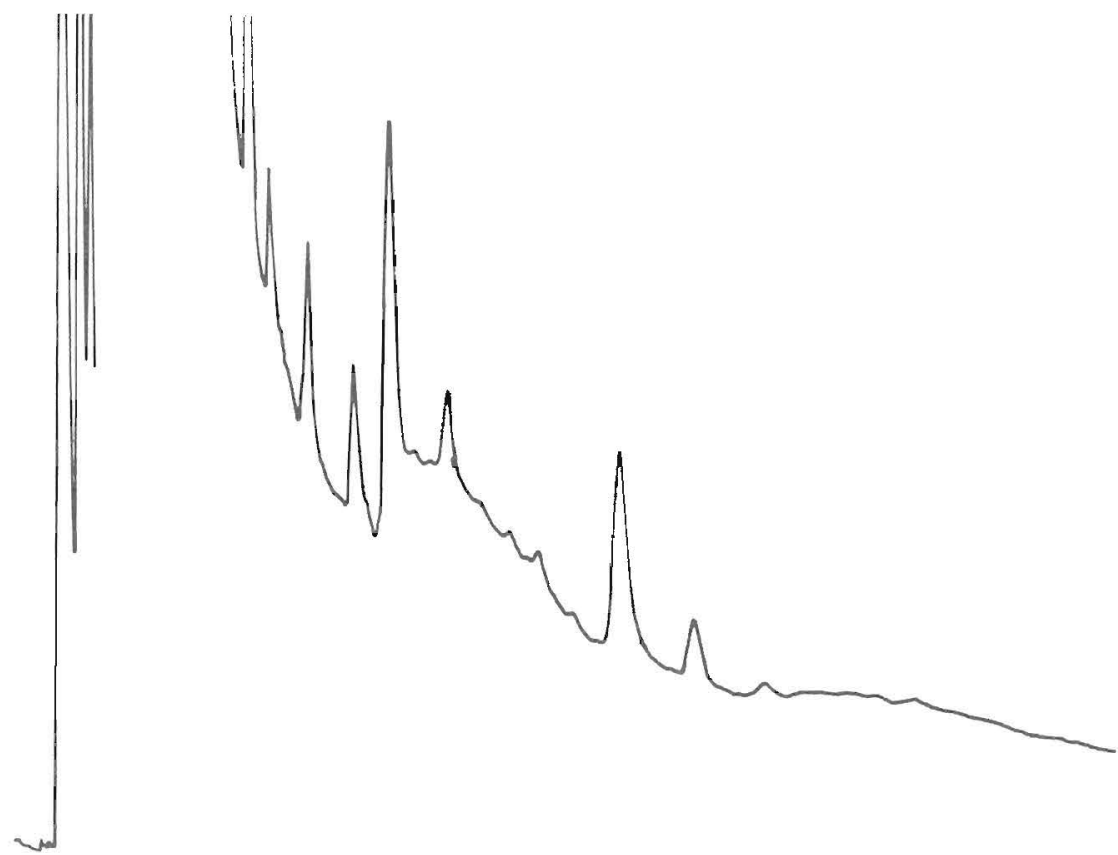

Fig. 4.-Chromatogram of R-701, $3 \mu \mathrm{l}$ in a high efficiency analytical packed column chromatogram; column PPR-132, $6.0 \mathrm{~m} \times 3.2 \mathrm{~mm}$ o.d. packed with acid washed, $60 / 80 \mathrm{~m}$ mesh Chromosorb W coated with $5 \%$ Carbowax 20M; carrier flow $25 \mathrm{ml} / \mathrm{min}$; initial temperature $58^{\circ} \mathrm{C}$ and final temperature $140^{\circ} \mathrm{C}$.

Identification of some congeners was accomplished by adding the respective component to the test sample. After positive identification, standard samples were prepared containing all components characterized, at concentrations similar to those found in the rums. Standard samples (P-31 and P-27) were separated in the same manner as the test samples and calibrations were made registering the retention times and areas with the integrator, whenever possible. The composition and retention times for the standard samples are given in table 1. The chromatogram for P- 
31 is reproduced in figure 1 and in figures 2 and 3 for P-27. The data for P-27 were taken with column PPR-130 operated at carrier flow of 12 $\mathrm{ml} / \mathrm{min}$, while those of P-31 were taken with column PPR-132 operated at $25 \mathrm{ml} / \mathrm{min}$. Chromatograms of $3 \mu \mathrm{l}$ samples of commercial rums ( $\mathrm{R}$ 703 and R-704) separated with the PPR-130 column at carrier flow of 12 $\mathrm{ml} / \mathrm{min}$, are reproduced in figures 2 and 3 together with a similar separation of standard sample P-27.

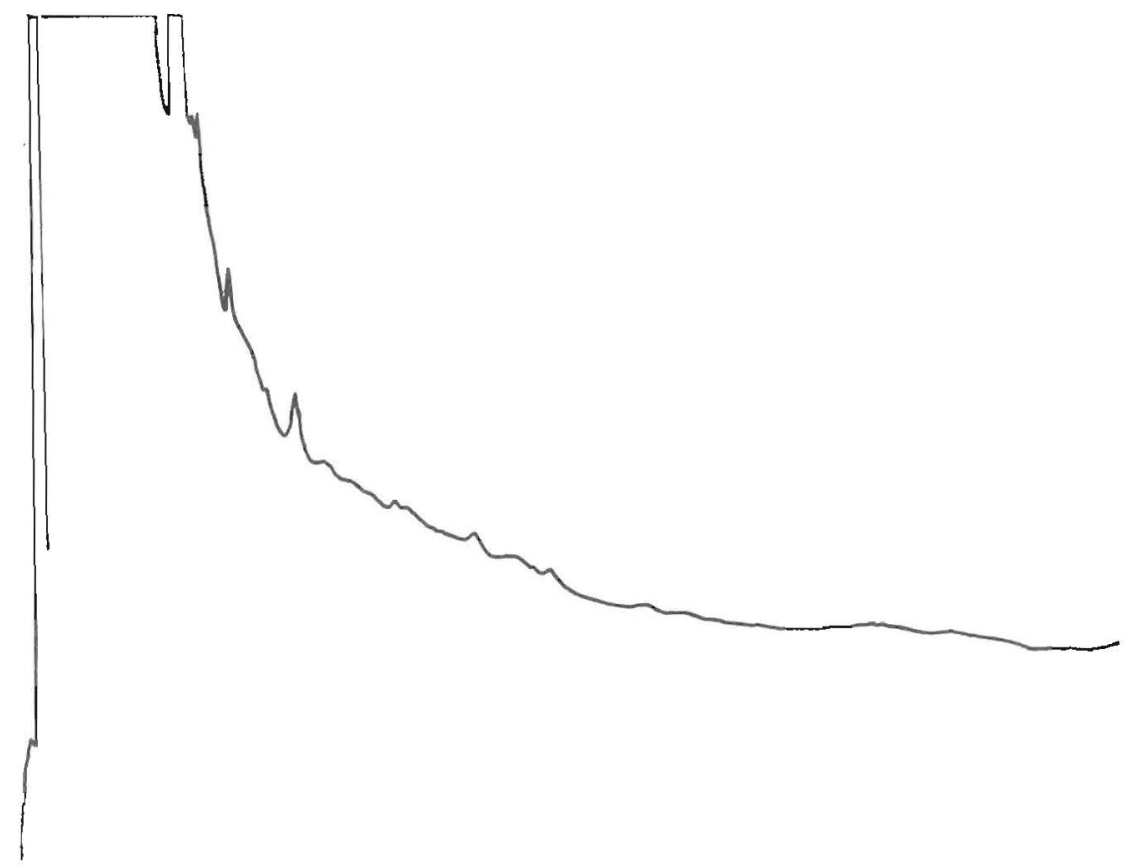

Fig. 5.-Chromatogram of R-702, $3 \mu \mathrm{l}$ in a high efficiency analytical packed column chromatogram; column PPR-132, $6.0 \mathrm{~m} \times 3.2 \mathrm{~mm}$ o.d. packed with acid washed, $60 / 80$ mesh Chromosorb W coated with $5 \%$ Carbowax 20M; carrier flow $25 \mathrm{ml} / \mathrm{min}$; initial temperature $58^{\circ} \mathrm{C}$ and final temperature $140^{\circ} \mathrm{C}$.

Chromatograms of typical commercial rums R-701, R-702, R-703, and R-704 are reproduced in figures 4, 5, 6, 7 for separated with the PPR-132 column operated at a carrier flow of $25 \mathrm{ml} / \mathrm{min}$. Quantitative determination for congeners so far identified are given in table 2 for all 1976 commercial rums. The calculation of the concentration of congeners separated after isoamyl alcohol, was based on at least three chromatograms of each rum obtained according to the method presented in figure 


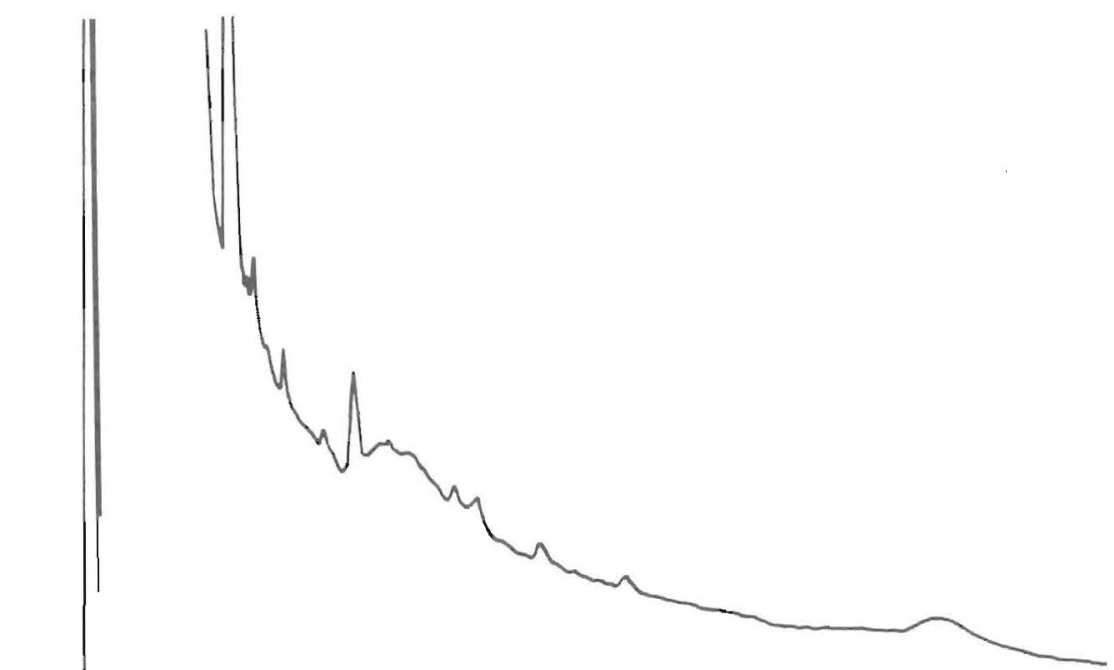

FIG. 6.-Chromatogram of R-703, $3 \mu \mathrm{l}$ in a high efficiency analytical packed column chromatogram; column PPR-132, $6.0 \mathrm{~m} \times 3.2 \mathrm{~mm}$ o.d. packed with acid washed, 60/80 mesh Chromosorb W coated with $5 \%$ Carbowax $20 \mathrm{M}$; carrier flow $25 \mathrm{ml} / \mathrm{min}$; initial temperature $58^{\circ} \mathrm{C}$ and final temperature $140^{\circ} \mathrm{C}$.
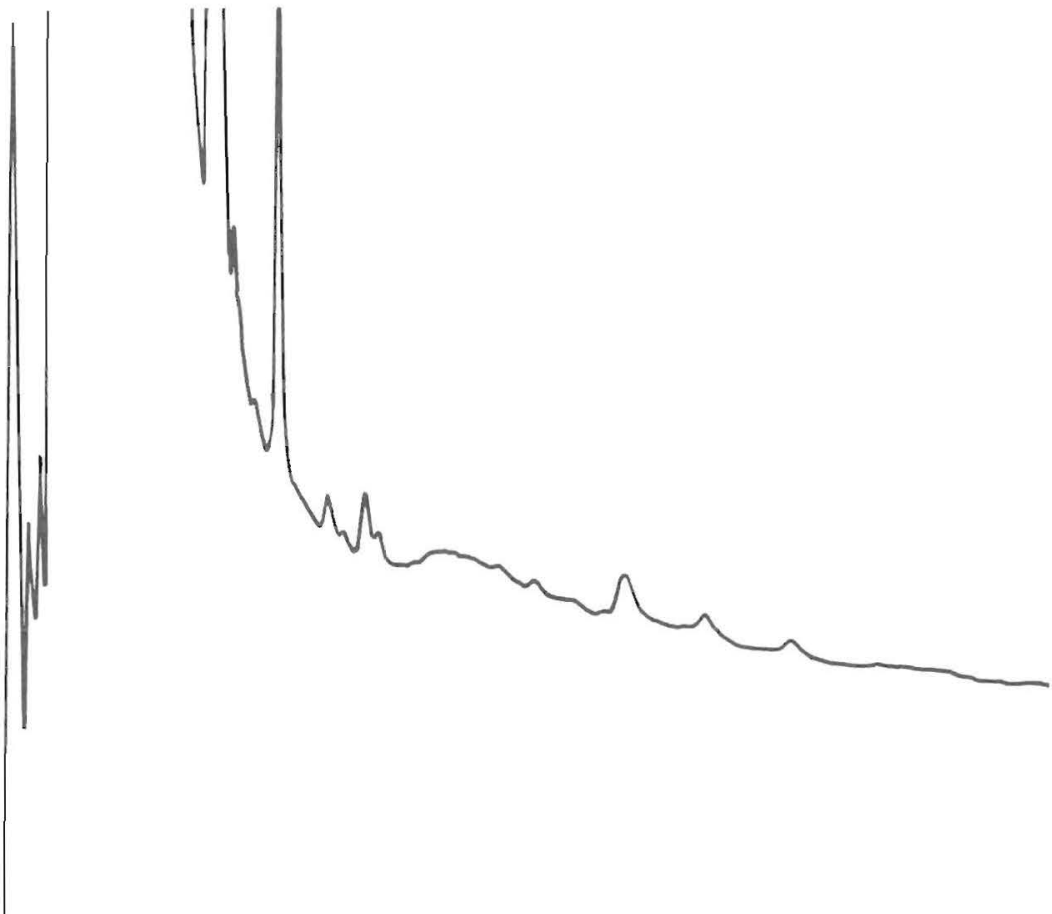

FIG. 7.-Chromatogram of R-704, $3 \mu \mathrm{l}$ in a high efficiency analytical packed column chromatogram; column PPR-132, $6.0 \mathrm{~m} \times 3.2 \mathrm{~mm}$ o.d. packed with acid washed, $60 / 80$ mesh Chromosorb W coated with $5 \%$ Carbowax $20 \mathrm{M}$; carrier flow $25 \mathrm{ml} / \mathrm{min}$; initial temperature $58^{\circ} \mathrm{C}$ and final temperature $140^{\circ} \mathrm{C}$. 
1. Those components separated before isoamyl alcohol, were calculated as in the method presented in figures 2 and 3. Estimates of the concentration of still unidentified congeners $(\mathrm{x})$ are also given in table 2, assuming that the area is related to the concentration of each signal.

TABLE 2.-Concentration of components identified in 1976 Puerto Rican commercial rums: concentration in $\mathrm{mg} / 100 \mathrm{ml} 80^{\circ} \mathrm{P}$

\begin{tabular}{|c|c|c|c|c|c|c|}
\hline Component & $\mathrm{R}-693$ & $\mathrm{R}-693$ & R-694 & R-695 & R-696 & $\mathrm{R}-697$ \\
\hline 1. Acetaldehyde & 6.6 & 6.6 & 6.7 & 6.5 & 6.6 & 0.7 \\
\hline 2. Methyl acetate & 1.2 & 2.8 & 2.8 & 2.7 & 2.7 & .3 \\
\hline 3. $\mathrm{X}^{1}$ & .2 & .2 & .2 & .2 & .2 & .1 \\
\hline 4. & 1.5 & 1.5 & 1.5 & 1.5 & 1.5 & .1 \\
\hline 5. $\quad \mathrm{X}$ & - & - & - & - & - & - \\
\hline 6. Ethyl acetate & 48.5 & 51.3 & 56.8 & 55.3 & 54.2 & 4.2 \\
\hline 7. Acetal & 3.3 & 3.5 & 3.5 & 3.2 & 3.4 & .6 \\
\hline 8. Ethyl alcohol & - & - & - & - & - & - \\
\hline 9. Propanol & 19.3 & 20.7 & 20.9 & 21.0 & 20.8 & 5.7 \\
\hline 10. Isobutyl alcohol & 2.5 & 2.5 & 2.6 & 2.5 & 2.4 & 3.0 \\
\hline 11. Isoamyl acetate & .1 & .1 & .1 & .1 & .1 & .1 \\
\hline 12. Butyl alcohol & - & - & - & - & - & .2 \\
\hline 13. $\quad \mathrm{X}$ & - & - & - & $\ldots$ & - & .1 \\
\hline 14. Isoamyl alcohol & 6.0 & 6.3 & 6.8 & 6.7 & 6.5 & 11.6 \\
\hline 15. Ethyl caproate & - & - & - & - & $\ldots$ & - \\
\hline 16. $\quad \mathrm{X}$ & .38 & .38 & .40 & .35 & .39 & $t^{2}$ \\
\hline 17. & - & - & - & - & - & .30 \\
\hline 18. $\mathrm{X}$ & - & - & - & - & - & .31 \\
\hline 19. Hexanol & .12 & .14 & .12 & .13 & .12 & .07 \\
\hline 20. $\mathrm{X}$ & $\mathrm{t}$ & $\mathrm{t}$ & $\mathrm{t}$ & t & $\mathrm{t}$ & .01 \\
\hline 21. & .01 & .01 & .01 & .01 & .01 & - \\
\hline 22. $\quad \mathrm{X}$ & .05 & .05 & .05 & .05 & .05 & $\mathrm{t}$ \\
\hline 23. Ethyl caprilate & .03 & .03 & .04 & .04 & .04 & .04 \\
\hline 24. $\quad \mathrm{X}$ & $\mathrm{t}$ & t & t & $\mathrm{t}$ & $\mathrm{t}$ & - \\
\hline 25. Furfural & .19 & .18 & .19 & .20 & .18 & .55 \\
\hline 26. $\mathrm{X}$ & - & - & - & - & - & $\mathrm{t}$ \\
\hline 27. & - & - & - & - & - & $t$ \\
\hline 28. $\quad \mathrm{X}$ & $\mathrm{t}$ & t & t & $\mathrm{t}$ & $\mathrm{t}$ & $\mathrm{t}$ \\
\hline 29. Furfuryl acetate & $\mathrm{t}$ & $\mathrm{t}$ & $\uparrow$ & i & $i$ & - \\
\hline 30. Octanol & .04 & .04 & Ud &. & .14 & .05 \\
\hline 31. $\mathrm{X}$ & - & - & - & - & - & $\mathrm{t}$ \\
\hline 32. & $\mathrm{t}$ & $\mathrm{t}$ & $\mathrm{t}$ & t & $\mathrm{t}$ & $t$ \\
\hline 33. & .10 & .10 & .10 & .10 & .10 & .10 \\
\hline 34. & .10 & .10 & .10 & .10 & .10 & $t$ \\
\hline 35. $\quad \mathrm{X}$ & .10 & .10 & .10 & .10 & .10 & $\mathrm{t}$ \\
\hline 36. Ethyl caprate & .08 & .08 & .08 & .10 & .08 & - \\
\hline 37. $\quad \mathrm{X}$ & .10 & .10 & .10 & .10 & .10 & .10 \\
\hline 38. & .10 & .10 & .10 & .10 & .10 & - \\
\hline 39. $\mathrm{X}$ & - & - & - & - & - & - \\
\hline 40. Decanol & - & - & - & - & - & - \\
\hline 41. Ethyl laurate & - & - & - & - & - & - \\
\hline 42. $\mathrm{X}$ & - & - & - & - & - & - \\
\hline 43. & .10 & .10 & .10 & .10 & .10 & - \\
\hline
\end{tabular}




\section{DISCUSSION}

Analytical high-efficiency packed gas chromatographic columns based on Carbowax 20M as liquid phase have proven to be most versatile for analysis of distilled alcoholic beverages. The new columns prepared and evaluated are extremely stable, durable, sensitive, and reproducible. The

TABLE 2.-Continued

\begin{tabular}{|c|c|c|c|c|c|c|}
\hline Component & $\mathrm{R}-698$ & R-699 & R-700 & $\mathrm{R}-701$ & $\mathrm{R}-702$ & R-703 \\
\hline 1. Acetaldehyde & 3.5 & 7.9 & 17.4 & 31.0 & 14.2 & 3.3 \\
\hline 2. Methyl acetate & .7 & 2.0 & 3.5 & 10.5 & 3.5 & .7 \\
\hline 3. $\mathrm{X}$ & .2 & $\mathrm{t}$ & .1 & - & $\mathrm{t}$ & .1 \\
\hline $\mathrm{x}$ & .1 & 1.2 & 1.7 & 1.5 & .5 & .1 \\
\hline 5. $\quad \mathrm{X}$ & .1 & - & - & - & .5 & .1 \\
\hline 6. Ethyl acetate & 10.9 & 32.0 & 19.8 & 49.8 & 37.3 & 9.9 \\
\hline 7. Acetal & 1.3 & 2.6 & 5.4 & - & - & 1.9 \\
\hline 8. Ethyl alcohol & - & - & - & - & - & - \\
\hline 9. Propanol & 5.9 & 11.2 & 4.5 & 45.1 & 3.3 & 8.3 \\
\hline 10. Isobutyl alcohol & 3.4 & 3.5 & 1.1 & 4.2 & 2.2 & 3.9 \\
\hline 11. Isoamyl acetate & $\mathrm{t}$ & $t$ & .1 & .1 & .1 & $\mathrm{t}$ \\
\hline 12. Butyl alcohol & .2 & .2 & .1 & .1 & .2 & .1 \\
\hline 13. $\mathrm{X}$ & - & - & $\mathrm{t}$ &. $\mathrm{I}$ & .3 & - \\
\hline 14. Isoamyl alcohol & 12.4 & 17.0 & .3 & 3.3 & 6.7 & 10.6 \\
\hline 15. Ethyl caproate & - & .01 & - & - & - & .01 \\
\hline 16. $\quad \mathrm{X}$ & .25 & .4 & .40 & .05 & .02 & .08 \\
\hline 17. & $t$ & $\mathrm{t}$ & - & .01 & .25 & .06 \\
\hline 18. $\mathrm{X}$ & t & .02 & t & t & .01 & t \\
\hline 19. Hexanol & .07 & .15 & .20 & .51 & .11 & .16 \\
\hline 20. $\mathrm{X}$ & .10 & .06 & - & .01 & $\mathrm{t}$ & .03 \\
\hline 21. & - & - & .06 & t & $\mathrm{t}$ & - \\
\hline 22. $\mathrm{X}$ & $\mathrm{t}$ & .14 & .15 & .70 & .10 & .06 \\
\hline 23. Ethyl caprilate & .03 & .03 & $\mathrm{t}$ & .01 & .01 & .06 \\
\hline 24. X & - & .04 & $\mathrm{t}$ & t & - & .02 \\
\hline 25. Furfural & .34 & .58 & .60 & 1.11 & .14 & .11 \\
\hline 26. $\mathrm{X}$ & t & - & - & $t$ & - & - \\
\hline 27. & $\mathrm{t}$ & $\mathrm{t}$ & t & .01 & $t$ & - \\
\hline 28. $\quad \mathrm{X}$ & $\mathrm{t}$ & t & t & $\mathrm{t}$ & .01 & - \\
\hline 29. Furfuryl acetate & t & - & - & t & .01 & - \\
\hline 30. Octanol & .04 & .05 & .14 & .45 & .01 & - \\
\hline 31. $\mathrm{X}$ & - & - & .01 & .02 & - & .01 \\
\hline 32. & t & .01 & - & .01 & .07 & - \\
\hline 33. & .01 & .07 & .07 & .10 & .10 & .10 \\
\hline 34. & - & .02 & .03 & .25 & - & - \\
\hline 35. $\quad \mathrm{X}$ & .01 & .06 & .06 & .10 & .09 & .08 \\
\hline 36. Ethyl caprate & .08 & $\mathrm{t}$ & - & - & $\mathrm{t}$ & - \\
\hline 37. $\quad \mathrm{X}$ & .01 & .04 & .55 & .85 & .08 & .13 \\
\hline 38. & - & .16 & .06 & .22 & - & .02 \\
\hline 39. $\mathrm{X}$ & - & .06 & - & .02 & - & - \\
\hline 40. Decanol & - & - & - & .04 & - & - \\
\hline 41. Ethyl laurate & - & - & - & - & - & - \\
\hline $42 . \quad \mathrm{X}$ & - & - & - & .60 & - & - \\
\hline 43. & - & - & - & - & - & - \\
\hline
\end{tabular}


principal result obtained with these columns, when operated at the unusually high carrier flow of $25 \mathrm{ml} / \mathrm{min}$, is that heavy trace rum congeners present in trace amounts in the beverage are readily separated. This is not the case with formerly-used techniques with which similar columns were used at low carrier flow due to lack of stability, and only

TABLE 2.-Continued

\begin{tabular}{|c|c|c|c|c|c|c|}
\hline Component & R-704 & $\mathrm{R}-705$ & $\mathrm{R}-706$ & $\mathrm{R}-707$ & $\mathrm{R}-708$ & R-709 \\
\hline 1. Acetaldehyde & 13.8 & 1.12 & 2.24 & 1.0 & 1.4 & 2.2 \\
\hline 2. Methyl acetate & 1.6 & .50 & .50 & .3 & .5 & 1.1 \\
\hline 3. $\quad \mathrm{X}$ & t & 一 & .1 & & - & \\
\hline 4. & 1.9 & $\mathrm{t}$ & .1 & & - & \\
\hline $5 . \quad \mathrm{X}$ & t & .1 & .1 & & - & \\
\hline 6. Ethyl acetate & 25.9 & 6.9 & 5.9 & 6.8 & 8.8 & 15.8 \\
\hline 7. Acetal & 4.8 & .5 & .6 & .7 & .8 & 2.3 \\
\hline 8. Ethyl alcohol & - & - & - & - & - & - \\
\hline 9. Propanol & 33.3 & 2.6 & 7.6 & 10.6 & 3.7 & 9.7 \\
\hline 10. Isobutyl alcohol & 14.3 & 1.6 & .8 & 1.1 & 2.5 & .6 \\
\hline 11. Isoamyl acetate & $\mathrm{t}$ & .1 & .3 & .1 & .2 & .4 \\
\hline 12. Butyl alcohol & .3 & .1 & 1 & - & .3 & .1 \\
\hline 13. $\mathrm{X}$ & & & & - & .1 & .1 \\
\hline 14. Isoamyl alcohol & 25.0 & 4.5 & 1.8 & & 16.2 & \\
\hline 15. Ethyl caproate & - & 一 & - & - & - & - \\
\hline 16. $\quad \mathrm{X}$ & .23 & .10 & .20 & .15 & .25 & .40 \\
\hline 17. & $\mathrm{t}$ & $\mathrm{t}$ & - & $\mathrm{t}$ & - & - \\
\hline 18. $\mathrm{X}$ & .01 & $\mathrm{t}$ & t & t & $\mathrm{t}$ & $\mathrm{t}$ \\
\hline 19. Hexanol & 1.01 & .06 & .06 & .03 & & .10 \\
\hline 20. X & .01 & - & .06 & .04 & .03 & .04 \\
\hline 21. & - & .02 & - & - & - & $\mathrm{t}$ \\
\hline 22. $\quad \mathrm{X}$ & .13 & .07 & .05 & .02 & .03 & .07 \\
\hline 23. Ethyl caprilate & .13 & .01 & - & - & $\mathrm{t}$ & .02 \\
\hline 24. $\quad \mathrm{X}$ & $\mathrm{t}$ & - & - & - & - & $\mathrm{t}$ \\
\hline 25. Furfural & .14 & .21 & .14 & .21 & .28 & .33 \\
\hline 26. $\mathrm{X}$ & - & t & - & - & - & - \\
\hline 27. & - & .05 & $\mathrm{t}$ & $t$ & - & $\mathrm{t}$ \\
\hline 28. $\quad \mathrm{X}$ & - & t & .02 & $\mathrm{t}$ & - & - \\
\hline 29. Furfuryl acetate & t & - & - & - & - & - \\
\hline 30. Octanol & .07 & 114 & .09 & .03 & .04 & .03 \\
\hline 31. $\mathrm{X}$ & i & $\mathrm{t}$ & .01 & - & - & - \\
\hline 32. & .01 & .01 & - & - & .01 & .07 \\
\hline 33. & .06 & .12 & .10 & .09 & .08 & .11 \\
\hline 34. & .05 & .06 & - & $\uparrow$ & - & .02 \\
\hline 35. $\quad \mathrm{X}$ & .05 & $.0 i$ & .08 & .04 & .06 & .0 \\
\hline 36. Ethyl caprate & .30 & - & - & - & - & - \\
\hline 37. $\mathrm{X}$ & .40 & 10.00 & .13 & 1.40 & .65 & .12 \\
\hline 38. & .10 & .05 & .02 & - & - & 0 \\
\hline 39. $\mathrm{X}$ & $\mathrm{t}$ & .07 & - & - & - & - \\
\hline 40. Decanol & $t$ & - & - & - & - & .03 \\
\hline 41. Ethyl laurate & - & - & - & - & - & - \\
\hline 42. $\mathrm{X}$ & .06 & - & - & - & - & - \\
\hline 43. & .02 & - & - & - & - & - \\
\hline
\end{tabular}


major volatile congeners could be separated, similar to those of figures 2 and 3. The columns developed for analysis of high-boiling congeners, were also used to advantage for the separation of low-boiling congeners at a carrier flow of $12 \mathrm{ml} / \mathrm{min}$.

TABLE 2.-Continued

\begin{tabular}{|c|c|c|c|c|c|c|}
\hline Component & $\mathrm{R}-710$ & $\mathrm{R}-711$ & $\mathrm{R}-712$ & R-714 & $\mathrm{R}-715$ & R-716 \\
\hline 1. Acetaldehyde & 2.3 & 1.5 & 5.6 & 2.6 & 3.2 & 2.4 \\
\hline 2. Methyl acetate & .7 & .2 & .7 & .7 & .6 & .7 \\
\hline 3. $\quad \mathrm{X}$ & .1 & $\mathrm{t}$ & $\mathrm{t}$ & & $t$ & .1 \\
\hline $\mathrm{X}$ & 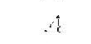 & .6 & .7 & & .3 & .2 \\
\hline 5. $\quad \mathrm{X}$ & $\mathrm{t}$ & - & - & & $\mathrm{t}$ & .1 \\
\hline 6. Ethyl acetate & 13.5 & 15.2 & 30.7 & 6.8 & 6.4 & 9.3 \\
\hline 7. Acetal & 1.7 & 1.5 & 2.6 & 1.4 & .2 & .1 \\
\hline 8. Ethyl alcohol & - & - & - & - & - & - \\
\hline Propanol & 10.7 & 21.4 & 21.5 & 4.1 & 8.0 & 16.0 \\
\hline 10. Isobutyl alcohol & 1.4 & 7.7 & 1.7 & .1 & .8 & 4.2 \\
\hline 11. Isoamyl acetate & .1 & 3.6 & I & .1 & .1 & 1.8 \\
\hline 12. Butyl alcohol & - & - & $\ldots$ & $\mathrm{t}$ & - & - \\
\hline 13. $\mathrm{X}$ & - & - & - & - & - & - \\
\hline 14. Isoamyl alcohol & 3.0 & 2.2 & 3.3 & .7 & 1.1 & .8 \\
\hline 15. Ethyl caproate & - & & - & - & - & - \\
\hline 16. $\quad \mathrm{X}$ & .17 & .25 & .27 & - & - & - \\
\hline 17. & - & - & - & - & - & - \\
\hline 18. $\mathrm{X}$ & .07 & .16 & i & - & - & - \\
\hline 19. Hexanol & .11 & 25 & .07 & - & .06 & .05 \\
\hline 20. X & .03 & .05 & .04 & .04 & - & - \\
\hline 21. & - & - & - & - & - & - \\
\hline 22. $\quad \mathrm{X}$ & .05 & .10 & .06 & $\mathrm{t}$ & - & - \\
\hline 23. Ethyl caprilate & .02 & .02 & .03 & - & - & - \\
\hline 24. $\quad \mathrm{X}$ & t & - & $\mathrm{t}$ & - & - & - \\
\hline 25. Furfural & .33 & 1.07 & .19 & .01 & .02 & .02 \\
\hline 26. $\mathrm{X}$ & - & - & $\mathrm{t}$ & - & - & - \\
\hline 27. & .01 & t & $\mathrm{t}$ & - & 一 & - \\
\hline 28. $\quad \mathrm{X}$ & t & $\mathrm{t}$ & $\mathrm{t}$ & - & - & - \\
\hline 29. Furfuryl acetate & $\mathrm{t}$ & - & $\mathrm{t}$ & - & - & - \\
\hline 30. Octanol & .04 & .10 & .02 & .01 & .02 & .02 \\
\hline 31. $\mathrm{X}$ & $t$ & $\mathrm{t}$ & - & - & - & - \\
\hline 32. & $\mathrm{t}$ & $\mathrm{t}$ & $.0 \mathrm{i}$ & t & - & - \\
\hline 33. & .05 & .13 & .08 & .02 & .02 & .02 \\
\hline 34. & .01 & .02 & .01 & $\mathrm{t}$ & $\mathrm{t}$ & $t$ \\
\hline 35. $\quad \mathrm{X}$ & .02 & .06 & .04 & t & - & - \\
\hline 36. Ethyl caprate & - & .10 & - & - & - & - \\
\hline 37. $\quad \mathrm{X}$ & .06 & .10 & .02 & - & - & - \\
\hline 38. & - & .08 & .04 & - & - & - \\
\hline 39. $\mathrm{X}$ & - & - & - & - & - & - \\
\hline 40. Decanol & - & - & - & - & - & - \\
\hline 41. Ethyl laurate & - & .06 & - & - & - & - \\
\hline 42. $\quad \mathrm{X}$ & - & - & - & - & - & - \\
\hline 43. & - & - & - & - & - & - \\
\hline
\end{tabular}

' $\mathrm{X}$, unknown

${ }^{2} t$, traces 
Twenty-three new congeners were separated: of these, eight were properly identified and are shown in table 1. These are: ethyl caproate, hexanol, ethyl caprylate, furfural, furfuryl acetate, octanol, ethyl caprate, and ethyl laureate, which are important congeners contributing to the aroma and/or the toxicity of the beverages.

The new columns are highly reproducible, but if they are not properly cleaned, portions of heavy components such as furfural and furfuryl acetate, when present at relatively high concentrations, remained in the column and, thus, appear later in the chromatogram of the sample injected afterward. This possibility is voided by multiple successive injections of spectral grade $80^{\circ} \mathrm{P}$ ethanol, which cleans the column and the injection system of the chromatograph. For most samples, with minor amounts of troublesome congeners, the cleaning procedure may be eliminated with no loss in reproducibility.

The new technique was applied for a full analysis of 1976 commercial Puerto Rican rums. The results summarized in table 2 show striking differences among the rums analyzed. Although many of the 23 new congeners have not been identified so far, this development is very useful for qualitative identification of rums or distilled alcoholic beverages, for scanning purposes when a particular component is sought, and for a rather good comprehensive analysis of distilled alcoholic beverages, such as that given in table 3 .

\section{RESUMEN}

Se describe un método simple de un solo paso, basado en cromatografia de vapor líquido, para la identificación y la determinación cuantitativa de congéneres en rones de Puerto Rico que no han sido informados anteriormente. Este método utiliza columnas de alta eficiencia de empaque, con lo que se logra la separación de unos 23 nuevos congéneres. La misma columna, cuando se opera a dos flujos distintos, puede separar rutinariamente más de 43 congéneres en los rones de Puerto Rico. De esta manera se analizaron muestras de todos los rones comerciales de Puerto Rico de 1976.

\section{LITERATURE CITED}

1. Batiz, H., and Soltero, E., 1976. Preparative-capillary gas chromatographic systems for trace analysis of rums, J. Agric. Univ. P.R. 60(4): 559-83.

2. Bober, A., and Haddaway, L. W., 1963. Gas chromatographic identification of alcoholic beverages, J. Gas Chromatogr., 1: 8-13.

3. Brunelle, R. L., 1968. Gas-liquid chromatography of fusel oils and ethyl acetate in spirits, J. AOAC, 5I(4): 915-21.

4. Kahn, J. H., Trent, F. M., Shipley, P. H., and Vordenberg, K. A., 1968. Gas chromatography of fusel oils in alcoholic distillates, J. AOAC, 51(6): 1330-3.

5. Suomalainen, H., and Nykanen, L., 1968. Methods applied in study of aroma of alcoholic beverages, Wallerstein Lab. Commun., 31: 5-13.

6. William, A. A., and Tucnott, O. F., 1972. The volatile components of scotch whiskey, J. Sci. Food Agric., 23: 1-7. 